\title{
GZK Neutrinos after the Fermi-LAT Diffuse Photon Flux Measurement
}

\author{
M. Ahlers ${ }^{\mathrm{a}}$, L. A. Anchordoqui ${ }^{\mathrm{b}}$, M. C. Gonzalez-Garcia ${ }^{\mathrm{a}, \mathrm{c}}$, F. Halzen ${ }^{\mathrm{d}}$, S. Sarkar ${ }^{\mathrm{e}}$ \\ ${ }^{a}$ C.N. Yang Institute for Theoretical Physics, SUNY at Stony Brook, Stony Brook, NY 11794-3840, USA \\ ${ }^{b}$ Department of Physics, University of Wisconsin-Milwaukee, Milwaukee, WI 53201, USA \\ ${ }^{c}$ Institució Catalana de Recerca i Estudis Avançats (ICREA), Departament d'Estructura i Constituents de la Matèria and \\ ICC-UB, Universitat de Barcelona, 647 Diagonal, E-08028 Barcelona, Spain \\ ${ }^{d}$ Department of Physics, University of Wisconsin, Madison, WI 53706, USA \\ ${ }^{e}$ Rudolf Peierls Centre for Theoretical Physics, University of Oxford, Oxford OX1 3NP, UK
}

\begin{abstract}
Cosmogenic neutrinos originate from photo-hadronic interactions of cosmic ray protons with the cosmic microwave background $(\mathrm{CMB})$. The neutrino production rate can be constrained through the accompanying electrons, positrons and $\gamma$-rays that quickly cascade on the CMB and intergalactic magnetic fields to lower energies and generate a $\gamma$-ray background in the $\mathrm{GeV}-\mathrm{TeV}$ region. Bethe-Heitler pair production by protons also contributes to the cascade and can tighten the neutrino constraints in models where extragalactic cosmic rays begin to dominate over the galactic component at a relatively low "crossover" energy. We investigate this issue in the light of the recent Fermi-LAT measurements of the diffuse extragalactic $\gamma$-ray background and illustrate by a fit to the HiRes spectrum how the prediction of the cosmogenic neutrino flux in all-proton models varies with the crossover energy. The neutrino flux is required to be smaller when the $\gamma$-ray bound is applied, nevertheless such models are still consistent with HiRes and Fermi-LAT if one properly takes into account the energy uncertainty of cosmic ray measurements. The presently allowed flux is within reach of the IceCube neutrino telescope and other dedicated radio experiments.
\end{abstract}

Keywords: cosmogenic neutrinos, low crossover model, diffuse gamma ray flux

Email addresses: ahlers@insti.physics.sunysb.edu (M. Ahlers), doqui@gravity.phys.uwm.edu (L. A. Anchordoqui), concha@insti.physics.sunysb.edu (M. C. Gonzalez-Garcia), halzen@icecube.wisc.edu (F. Halzen), s.sarkar@physics.ox.ac.uk (S. Sarkar) 


\section{Introduction}

Soon after the discovery of the cosmic microwave background (CMB) 1], it was realized that interactions of extragalactic ultrahigh energy (UHE) cosmic rays (CRs) on the relic photons would suppress the cosmic ray flux at energies $\gtrsim 5 \times 10^{10} \mathrm{GeV}$, the so-called "GZK cutoff" [2, 3]. It was pointed out subsequently [4] that the GZK interaction also generates a "cosmogenic flux" of neutrinos, through the decay of secondary charged pions. Forty years later, the predicted suppression of the UHE CR flux was indeed observed by the HiRes [5] and Auger [6] experiments. However, the cosmogenic flux of neutrinos has yet to be detected.

The GZK reaction chain generating cosmogenic neutrinos is well known [7]. The intermediate state of the reaction $p \gamma_{\mathrm{CMB}} \rightarrow n \pi^{+} / p \pi^{0}$ is dominated by the $\Delta^{+}$resonance, because the neutron decay length is smaller than the nucleon mean free path on the CMB. Resonant $p \gamma$ interactions produce twice as many neutral pions as charged pions. Direct pion production via virtual meson exchange contributes only about $20 \%$ to the total cross-section, but is almost exclusively into $\pi^{+}$. Hence, $p \gamma$ interactions produce roughly equal number of $\pi^{+}$and $\pi^{0}$. Gamma-rays, produced via $\pi^{0}$ decay, subsequently cascade electromagnetically on intergalactic radiation fields through $e^{+} e^{-}$pair production followed by inverse Compton scattering. The net result is a pile up of $\gamma$-rays at $\mathrm{GeV}-\mathrm{TeV}$ energies, just below the threshold for further pair production on the diffuse optical background. Meanwhile each $\pi^{+}$decays to 3 neutrinos and a positron; the $e^{+}$readily loses its energy through inverse Compton scattering on the diffuse radio background or through synchrotron radiation in intergalactic magnetic fields. The neutrinos carry away about $3 / 4$ of the $\pi^{+}$energy, therefore the energy in cosmogenic neutrinos is about $3 / 4$ of that produced in $\gamma$-rays.

The normalization of the neutrino flux depends critically on the cosmological evolution of the CR sources and on their proton injection spectra [8]. It also depends on the assumed spatial distribution of sources; for example, local sources in the Virgo cluster 9, would dominate the high energy tail of the proton spectrum. Another source of uncertainty is the energy at which there is a transition from Galactic to extragalactic CRs as inferred from a change in the spectral slope. The "ankle" at $\sim 3 \times 10^{9} \mathrm{GeV}$ seems to be a natural candidate for this transition [10, 11, 12, but a lower energy crossover at the "second knee" at $\sim 5 \times 10^{8} \mathrm{GeV}$ has also been advocated [13, 14. A fourth source of uncertainty is the chemical composition of the parent CRs - if these are heavy nuclei rather than protons, then the neutrino flux is reduced [15.

The most up-to-date calculation [16] of the cosmogenic neutrino flux combines a double-fit analysis of the energy [17] and elongation rate [18] measurements to constrain the spectrum and chemical composition of UHE CRs at their sources. Injection models with a wide range of chemical compositions are found to be consistent with observations. The data is consistent with a proton-dominated spectrum with a small admixture of heavy nuclei, in which case the cosmogenic neutrino flux is rather similar to the all-proton model. In this case, kilometer-scale neutrino telescopes are expected to observe of $\mathcal{O}(1)$ cosmogenic neutrino event per year. In contrast, an intermediate to heavy nuclear composition beyond the ankle, as indicated by the elongation rate vs. energy [18, can lead to a considerable suppression (up to two orders of magnitude) of the cosmogenic neutrino flux in comparison to the all-proton case. However, little is known about the chemical composition from just below to beyond the GZK cutoff, where the most significant contribution to cosmogenic neutrinos form UHE CR protons is expected. It is interesting to note that uncertainties in the extrapolation of the proton-air interaction - cross-section, elasticity and multiplicity of secondaries - from accelerator measurements to the high energies characteristic for air showers are large enough to undermine any definite conclusion on the chemical composition [19, 20.

In this work we study the constraint set by the diffuse $\gamma$-ray background on all-proton models of extragalactic CRs. We parametrize our ignorance of the crossover energy - which marks the transition between the galactic and extragalactic components - as a variable low energy cutoff in the proton injection rate. By fitting only to CR data above the crossover energy, taken to be between $10^{17.5} \mathrm{eV}$ and $10^{19} \mathrm{eV}$, we determine the statistically preferred values of the spectral index $\gamma$ and cosmic source density evolution index $n$ by a goodness-of-fit (GOF) test of the HiRes data, taking into account the energy resolution of about $25 \%$. For each model we check that the total energy density of the EM cascade is below a critical value inferred from the recent measurement of the extragalactic $\gamma$-ray background by the Fermi-LAT Collaboration [21. We 
find that the allowed range of the cosmogenic neutrino flux increases with the crossover energy and can be up to an order of magnitude larger than the values presented in a recent study [22].

This paper is organized as follows. We begin in $\S 2$ with a discussion of the extragalactic proton fluxe and the corresponding energy density of cascade $\gamma$-rays. We present our statistical method in $\S 3$ and discuss our results in $\S 4$

\section{Extra-galactic Proton Fluxes and Diffuse Gamma Background}

For a spatially homogeneous distribution of cosmic sources, emitting UHE particles of type $i$, the comoving number density $Y_{i}$ is governed by a set of (Boltzmann) continuity equations of the form:

$$
\dot{Y}_{i}=\partial_{E}\left(H E Y_{i}\right)+\partial_{E}\left(b_{i} Y_{i}\right)-\Gamma_{i} Y_{i}+\sum_{j} \int \mathrm{d} E_{j} \gamma_{j i} Y_{j}+\mathcal{L}_{i},
$$

together with the Friedman-Lemaître equations describing the cosmic expansion rate $H(z)$ as a function of the redshift $z 1^{1}$ The first and second terms on the r.h.s. describe, respectively, redshift and other continuous energy losses (CEL) with rate $b \equiv \mathrm{d} E / \mathrm{d} t$. The third and fourth terms describe more general interactions involving particle losses $\left(i \rightarrow\right.$ anything) with interaction rate $\Gamma_{i}$, and particle generation of the form $j \rightarrow i$ with differential interaction rate $\gamma_{i j}$. The last term on the r.h.s., $\mathcal{L}_{i}$, corresponds to the emission rate per co-moving volume of CRs $i$. We refer to Ref. 24 for explicit definitions of the coefficients in Eq. (1).

Extragalactic protons lose their energy via Bethe-Heitler $(\mathrm{BH})$ pair production and photo-hadronic interactions on cosmic radiation backgrounds, notably the CMB. Bethe-Heitler pair production, $p+\gamma_{\mathrm{bgr}} \rightarrow$ $p+e^{+}+e^{-}$, can be treated as a continuous energy loss due to its low inelasticity [25]. This process dominates the evolution of the spectra at energies between $10^{9} \mathrm{GeV}$ and a few times $10^{10} \mathrm{GeV}$. At higher energies, resonant photo-hadronic interactions with CMB photons lead to a sharp suppression of the spectrum [2, 3]. The produced charged and neutral pions release electrons, positrons, neutrinos and photons through their decay. We calculate the spectra of hadrons and neutrinos using the Monte Carlo package SOPHIA [26].

It is possible to approximate the energy loss in the hadronic cascade due to photo-pion production as a CEL with

$$
\frac{\mathrm{d} E}{\mathrm{~d} t}(z, E) \equiv b(z, E) \simeq E \Gamma_{p}(z, E)-\int \mathrm{d} E^{\prime} E^{\prime} \gamma_{p p}\left(z, E, E^{\prime}\right)
$$

Diffractive $p \gamma$ processes at high energies with large final state multiplicities of neutrons and protons ultimately invalidate the CEL approximation. However, the relative error below $10^{12} \mathrm{GeV}$ is less than $15 \%$ so we will use this approximation for a detailed numerical scan in the model space of proton spectra.

Electromagnetic (EM) interactions of photons and leptons with the extragalactic radiation backgrounds and magnetic field can happen on time-scales much shorter than their production rates. The relevant processes with background photons contributing to the differential interaction rates $\gamma_{e e}, \gamma_{\gamma e}$ and $\gamma_{e \gamma}$ are inverse Compton scattering (ICS), $e^{ \pm}+\gamma_{\mathrm{bgr}} \rightarrow e^{ \pm}+\gamma$, pair production (PP), $\gamma+\gamma_{\mathrm{bgr}} \rightarrow e^{+}+e^{-}$, double pair production (DPP) $\gamma+\gamma_{\mathrm{bgr}} \rightarrow e^{+}+e^{-}+e^{+}+e^{-}$, and triple pair production (TPP), $e^{ \pm}+\gamma_{\mathrm{bgr}} \rightarrow$ $e^{ \pm}+e^{+}+e^{-}$[25, 27]. High energy electrons and positrons can also lose energy via synchrotron radiation on the intergalactic magnetic field ${ }^{2}$ the strength of which is limited to be below $\sim 10^{-9} \mathrm{G}[30$ ] and suggested to be of $\mathcal{O}\left(10^{-12}\right) \mathrm{G}$ by simulations of large-scale structure formation 32 ; details on the calculation are given in Appendix A

\footnotetext{
${ }^{1}$ This is given by $H^{2}(z)=H_{0}^{2}\left[\Omega_{\mathrm{m}}(1+z)^{3}+\Omega_{\Lambda}\right]$, normalised to its value today of $H_{0} \sim 70 \mathrm{~km} \mathrm{~s}^{-1} \mathrm{Mpc}^{-1}$, in the usual "concordance model" dominated by a cosmological constant with $\Omega_{\Lambda} \sim 0.7$ and a (cold) matter component, $\Omega_{\mathrm{m}} \sim 0.323$. The time-dependence of the redshift can be expressed via $\mathrm{d} z=-\mathrm{d} t(1+z) H$.

${ }^{2}$ Since we consider a relatively strong intergalactic magnetic field, we can neglect TPP by electrons in the following [28]. Also below $10^{12} \mathrm{GeV}$ we can safely neglect DPP of photons in the calculation [29].
} 
Synchrotron radiation in strong magnetic fields can also by-pass the EM cascade and transfer energy to sub-GeV photons that are unconstrained by the Fermi-LAT spectrum [31]. In the case of a strong $10^{-9} \mathrm{G}$ field this can be relevant for electrons around $10^{9} \mathrm{GeV}$, where synchrotron loss starts to dominate over ICS loss in the CMB and where the corresponding synchrotron spectrum still peaks below $100 \mathrm{MeV}$. In our calculation we adopt a moderate value of $10^{-12} \mathrm{G}$ following $\underline{32}$. However, we have checked that a significantly larger field strength of $10^{-9} \mathrm{G}$ has little effect on the $\gamma$-ray flux in the Fermi-LAT energy range relevant for our discussion (see Fig. C.8 in the Appendix).

In this paper the emission rate of CR protons per co-moving volume $\left(\mathrm{GeV}^{-1} \mathrm{~cm}^{-3} \mathrm{~s}^{-1}\right)$ is assumed, as per usual practice, to follow a power-law:

$$
\mathcal{L}_{p}(0, E) \propto\left(E / E_{0}\right)^{-\gamma} \times \begin{cases}f_{-}\left(E / E_{\min }\right) & E<E_{\min }, \\ 1 & E_{\min }<E<E_{\max } \\ f_{+}\left(E / E_{\max }\right) & E_{\max }<E .\end{cases}
$$

We will consider spectral indices $\gamma$ in the range $2 \div 3$. The functions $f_{ \pm}(x) \equiv x^{ \pm 2} \exp \left(1-x^{ \pm 2}\right)$ in Eq. (3) smoothly turn off the contribution below $E_{\min }$ and above $E_{\max }$. We set $E_{\max }=10^{21} \mathrm{eV}$ in the following and vary $E_{\min }$ in the range $10^{17.5} \div 10^{19} \mathrm{eV}$, corresponding to a galactic-extragalactic crossover between the "second knee" and the "ankle" in the CR spectrum.

The cosmic evolution of the spectral emission rate per comoving volume is parameterized as:

$$
\mathcal{L}_{p}(z, E)=\mathcal{H}(z) \mathcal{L}_{p}(0, E)
$$

For simplicity, we use the standard approximation

$$
\mathcal{H}(z) \equiv(1+z)^{n} \Theta\left(z_{\max }-z\right),
$$

with $z_{\max }=2$. Note that the dilution of the source density due to the Hubble expansion is taken care of since $\mathcal{L}$ is the comoving density, i.e. for no evolution we would simply have $\mathcal{H}=1$. We consider cosmic evolution of UHE CR sources with $n$ in the range $2 \div 6$.

As mentioned above, EM interactions of photons and leptons with the extra-galactic background light and magnetic field can happen on time-scales much shorter than their production rates. It is convenient to account for these contributions during the proton propagation as fast developing electro-magnetic cascades at a fixed redshift. We will use the efficient method of "matrix doubling" [33] for the calculation of the cascades. Since the cascade $\gamma$-ray flux is mainly in the $\mathrm{GeV}-\mathrm{TeV}$ region and has an almost universal shape here, it is numerically much more efficient to calculate the total energy density $\omega_{\text {cas }}$ injected into the cascade and compare this value to the limit imposed by Fermi-LAT. The total energy density $\left(\mathrm{eV} \mathrm{cm}^{-3}\right) \mathrm{of} \mathrm{EM}^{\mathrm{E}}$ radiation from proton propagation in the past is given as

$$
\omega_{\text {cas }} \equiv \int \mathrm{d} E E n_{\text {cas }}(0, E)=\int \mathrm{d} t \int \mathrm{d} E \frac{b_{\text {cas }}(z, E)}{(1+z)^{4}} n_{\mathrm{p}}(z, E)
$$

where $n(z, E)$ is the physical energy density at redshift $z$, defined via $n(z, E) \equiv(1+z)^{3} Y(z, E)$. We discuss the derivation of this equation in Appendix B. The continuous energy loss of protons into the cascade, denoted by $b_{\text {cas }}$, is in the form of electron, positron and $\gamma$-ray production in $\mathrm{BH}\left(b_{\mathrm{BH}}\right)$ and photo-pion $\left(b_{\pi}\right)$ interactions 3

In the following we derive the $\mathrm{BH}$ and photo-pion contribution to $\omega_{\text {cas }}$ separately. For the photo-pion contribution we estimate

$$
b_{\pi}(z, E) \simeq \int \mathrm{d} E^{\prime} E^{\prime}\left[\gamma_{p e^{-}}\left(z, E, E^{\prime}\right)+\gamma_{p e^{+}}\left(z, E, E^{\prime}\right)+\gamma_{p \gamma}\left(z, E, E^{\prime}\right)\right]
$$

\footnotetext{
${ }^{3}$ Note the difference between our Eq. $\sqrt{6}$ and Eq. (10) in Ref. 22$]$ where the approximation $\partial_{E} b(z, E) \simeq b(z, E) / E$ is used (cf. Fig. 5 in Ref. 24]) and an adiabatic scaling with redshift, $b(z, E) \simeq(1+z)^{2} b(0, E(1+z))$, is assumed.
} 

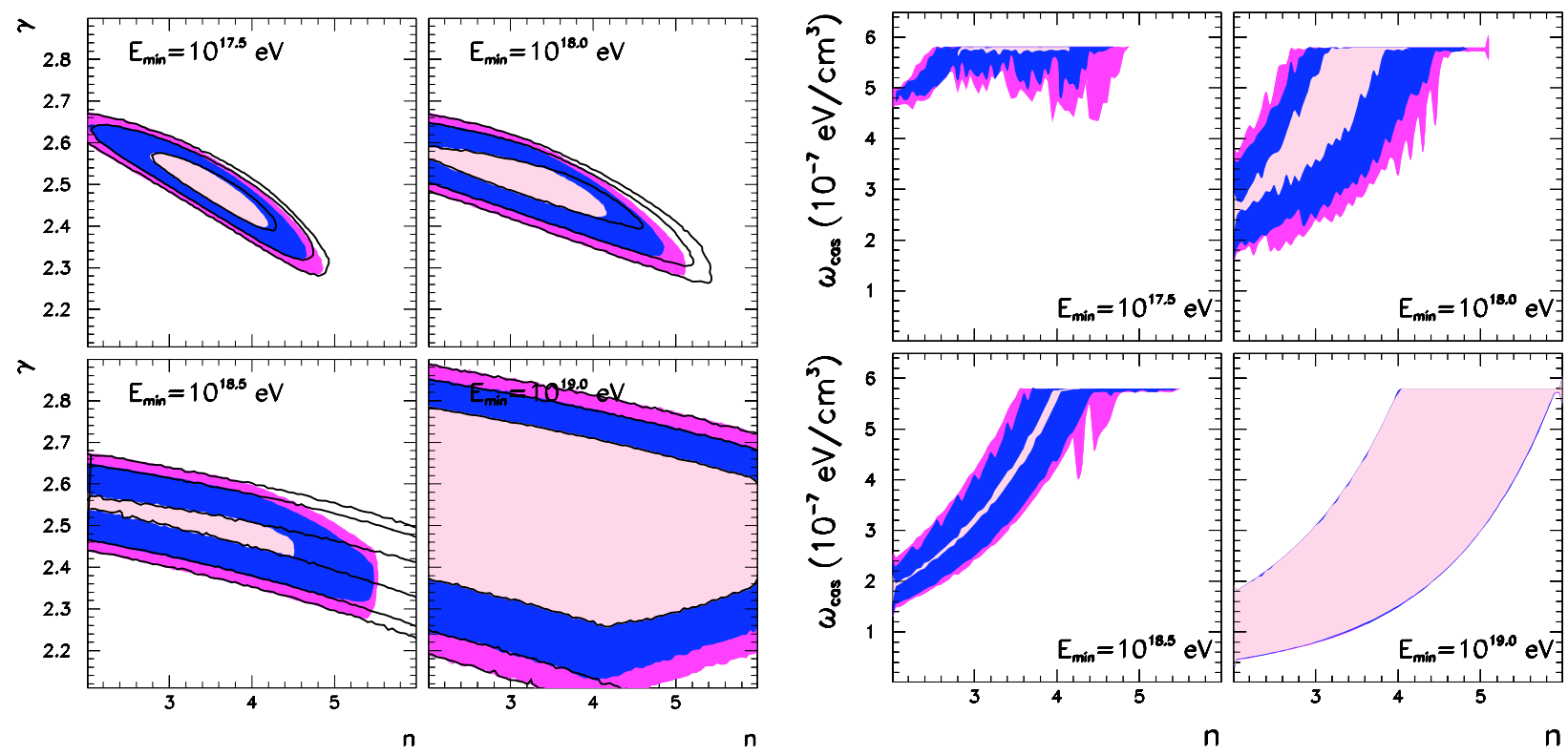

Figure 1: Left Panel: Goodness of fit test of the HiRes data 5]. We show the $68 \%$ (pink), 95\% (blue) and 99\% (magenta) confidence levels of the injection index $\gamma$ and the cosmic evolution index $n$. The black lines indicate the allowed regions before the cascade $\left(\omega_{\text {cas }}\right)$ bound is imposed. Right Panel: The corresponding energy density in the EM cascade.

For the energy loss via $\mathrm{BH}$ pair production we use the expression given in Ref. 25]. Note, that since the photo-pion contribution in the cascade is dominated at the GZK cutoff its contribution should increase relative to $\mathrm{BH}$ pair production with increasing crossover energy and, hence, also the associated neutrino fluxes after normalization to $\gamma$-ray and CR data.

\section{Goodness of Fit Test}

In this section we present the results of a GOF test of the compatibility of a given model, characterized by the injection spectral index $\gamma$, cosmic evolution index $n$, and crossover energy $E_{\min }$, with the CR experimental data - in particular HiRes I and II [5] - imposing also consistency with the Fermi-LAT measurements of the diffuse extra-galactic $\gamma$-ray background.

Given the acceptance $A_{i}$ (in units of area per unit time per unit solid angle) of the experiment for the energy bin $i$ centered at $E_{i}$ and with bin width $\Delta_{i}$, and the energy scale uncertainty of the experiment, $\sigma_{E_{s}}$ the number of expected events in the bin is given by

$$
N_{i}(n, \gamma, \mathcal{N}, \delta)=A_{i} \int_{E_{i}(1+\delta)-\Delta_{i} / 2}^{E_{i}(1+\delta)+\Delta_{i} / 2} J_{\mathcal{N}, n, \gamma}^{p}(E) d E
$$

where $J_{\mathcal{N}, n, \gamma}^{p}(E)=n_{p}(0, E) \frac{c}{4 \pi}$ is the proton flux arriving at the detector corresponding to a proton source luminosity as in Eq. (3), with the cosmic evolution of the source density given by Eqs. (4) and (5). The parameter $\delta$ in Eq. (8) above is a fractional energy-scale shift that reflects the energy-scale uncertainty of the experiment, and $\mathcal{N}$ is the normalization of the proton source luminosity.

The probability distribution of events in the $i$-th bin is of the Poisson form with mean $N_{i}$. Correspondingly the $r$-dimensional ( $r$ being the number of bins of the experiment with $E_{i} \geq E_{\min }$ ) probability 


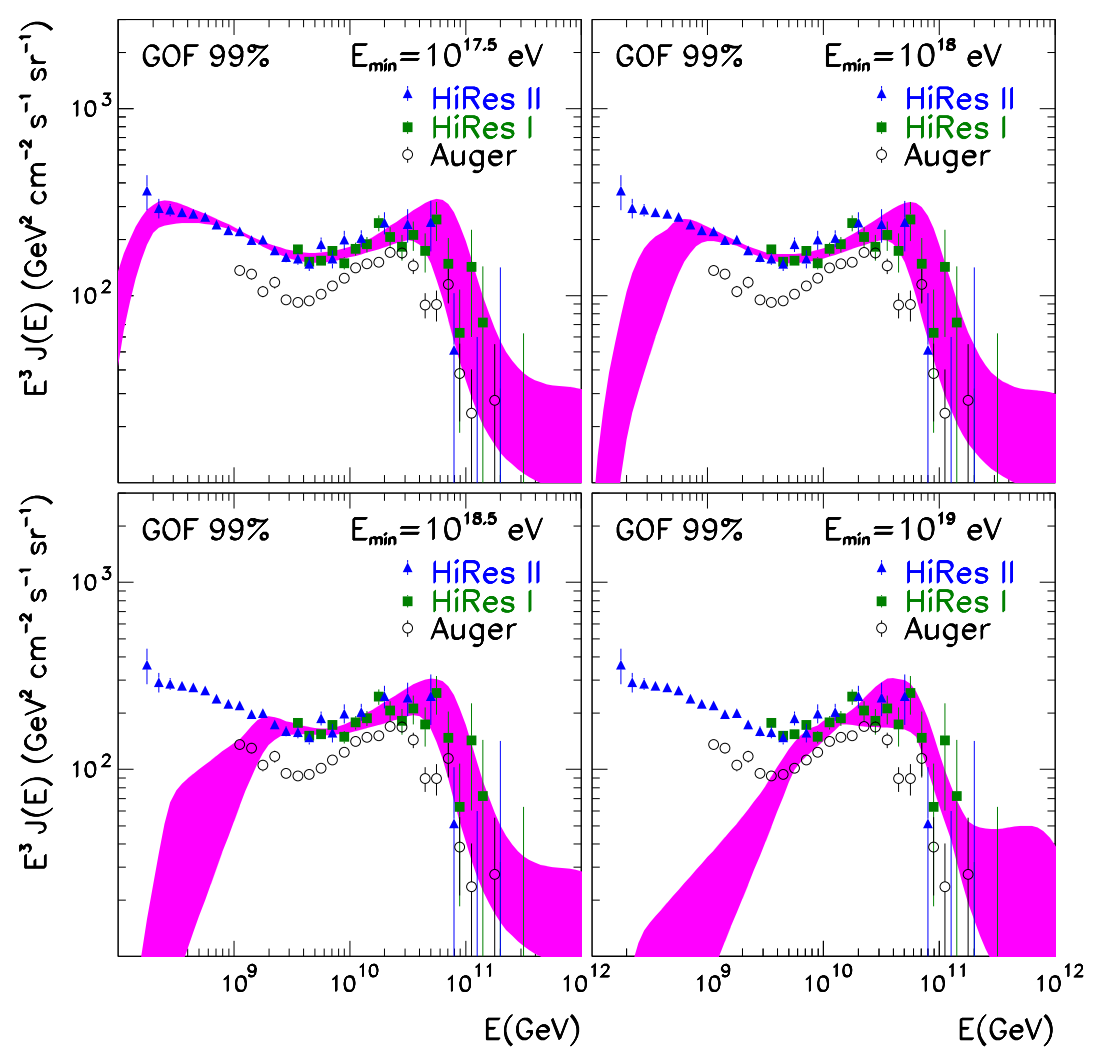

Figure 2: The allowed proton flux (at the $99 \%$ confidence level) for increasing crossover energy $E_{\text {min }}$. Each fit of the proton spectrum is marginalized with respect to the experimental energy uncertainty and we show the shifted predictions in comparison to the HiRes central values [5]. For comparison we also show the Auger data [6, 17] which has not been included in the fit.

distribution for a set of non-negative integer numbers $\vec{k}=\left\{k_{1}, \ldots k_{r}\right\}, P_{\vec{k}}(n, \gamma, \mathcal{N}, \delta)$, is just the product of the individual Poisson distributions.

According to this $r$-dimensional probability distribution, the experimental result $\vec{N}^{\exp }=\left\{N_{1}^{\exp }, \ldots, N_{r}^{\exp }\right\}$ has a probability $P_{\vec{N} \exp }(n, \gamma, \mathcal{N}, \delta)$ and correspondingly the experimental probability after marginalizing over the energy scale uncertainty and normalization is:

$$
P_{\exp }(n, \gamma)=\operatorname{Max}_{\delta, \mathcal{N}} P_{\vec{N} \exp }(n, \gamma, \mathcal{N}, \delta) .
$$

where the maximization is made within some prior for $\delta$ and $\mathcal{N}$. For the energy shift $\delta$ we have used two forms for the prior, either a top hat spanning the energy-scale uncertainty of the experiment, $\sigma_{E_{s}}$, or a gaussian prior of width $\sigma_{E_{s}}$.

For $\mathcal{N}$ we impose the prior arising from requiring consistency with the Fermi-LAT measurements [21] of the diffuse extra-galactic $\gamma$-ray background. In order to do so we obtain the total energy density of EM radiation from the proton propagation using Eq. (6) and we require following Ref. [22]:

$$
w_{\text {cas }}(\mathcal{N}, n, \gamma) \leq 5.8 \times 10^{-7} \mathrm{eV} / \mathrm{cm}^{3} .
$$

The marginalization in Eq. (9) also determines $\mathcal{N}_{\text {best }}$ and $\delta_{\text {best }}$ for the model, which are the values of the 


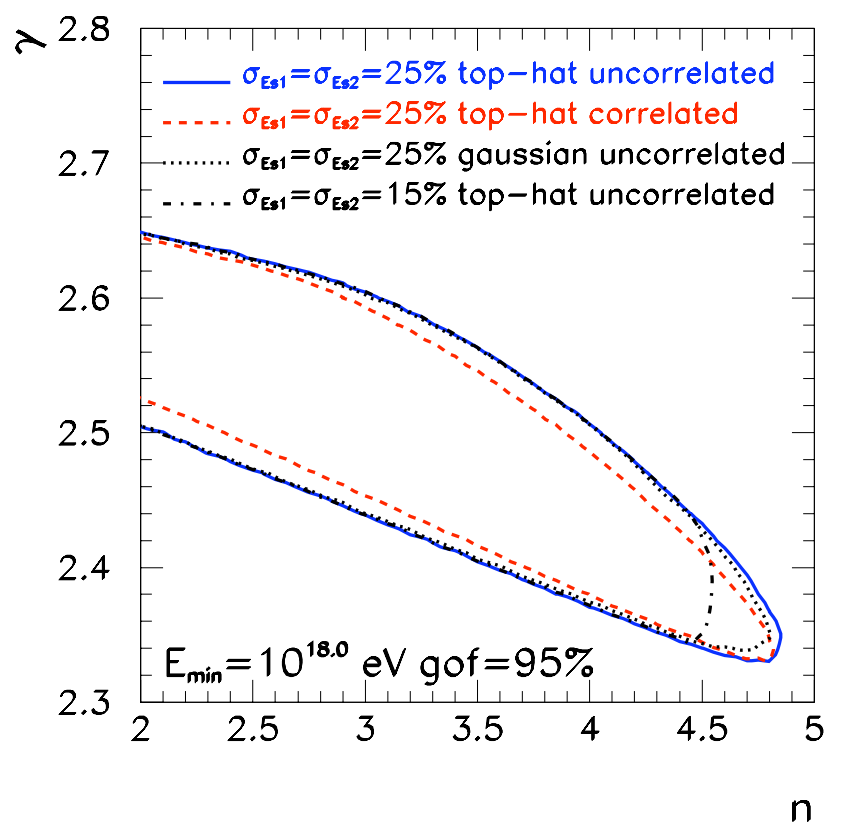

Figure 3: Systematic effect of the experimental energy resolution on the fitted spectral index $\gamma$ and cosmological evolution parameter $n$. For illustration we show the dependence of the $95 \%$ C.L. bound for a crossover energy of $10^{18}$ eV. The blue contour corresponds to the region shown in Fig. 1 assuming an uncorrelated energy shift of $25 \%$ in both data sets (HiRes I and II) 5, for a flat prior ("top-hat" distribution). The red dashed curve assumes correlated errors of the energy resolution in both data sets. The black dotted curve shows the result for uncorrelated errors with a Gaussian prior, and the dashed-dotted line shows uncorrelated errors with a flat prior, but with a lower uncertainty of $15 \%$.

energy shift and normalization that yield the best description of the experimental CR data, subject to the constraint imposed by the Fermi-LAT measurement.

Altogether the model is compatible with the experimental results at given goodness of the fit (GOF) if

$$
\sum_{\vec{k}} P_{\vec{k}}\left(n, \gamma, \mathcal{N}_{\text {best }}, \delta_{\text {best }}\right) \Theta\left[P_{\vec{k}}\left(n, \gamma, \mathcal{N}_{\text {best }}, \delta_{\text {best }}\right)-P_{\text {exp }}(n, \gamma)\right] \leq \mathrm{GOF}
$$

Technically, this is computed by generating a large number $N_{\text {rep }}$ of replica experiments according to the probability distribution $P_{\vec{k}}\left(n, \gamma, \mathcal{N}_{\text {best }}, \delta_{\text {best }}\right)$ and imposing the fraction $F$ of those which satisfy $P_{\vec{k}}\left(n, \gamma, \mathcal{N}, \delta_{\text {best }}\right)>$ $P_{\exp }(n, \gamma)$ to be $F \leq \mathrm{GOF}$.

With this method we determine the value of $(n, \gamma)$ parameters that are compatible with the HiRes I and HiRes II experiments [5]. We plot in the left panel of Fig. 1 the regions with GOF $64 \%, 95 \%$ and $99 \%$ for four values of the minimum (i.e. crossover) energy. In the right panel we show the corresponding ranges of $w_{\text {cas,best }}$ for the models as a function of the cosmic evolution index $n$. In order to display explicitly the impact of the constraint from the Fermi-LAT measurements of the diffuse extra-galactic $\gamma$-ray background (10), we show the corresponding GOF regions without imposing that constraint. In Table. 1 we list the parameters corresponding to the best-fit models and to the models with minimal and maximal contributions to $\omega_{\pi}$ and $\omega_{\text {cas }}=\omega_{\pi}+\omega_{\mathrm{BH}}$ at the $99 \%$ C.L., together with the corresponding energy shifts which give best fits to the HiRes I and Hires II data. We also show the parameters for the models with maximum $\omega_{\pi}$ and $\omega_{\text {cas }}$ without imposition of the Fermi-LAT constraint.

As an illustration of the agreement with the CR data we show in Fig. 1 the range of proton fluxes corresponding to models with GOF $99 \%$ or better for increasing crossover energies $E_{\min }$. As discussed above each fit of the proton spectra is marginalized with respect to the experimental energy scale uncertainty and we show the shifted predictions with $\delta_{\text {best }}$ in comparison to the HiRes data at central value. We also show in the figure the results from Auger [6, 17, though these have not been included in the analysis (see below). 


\begin{tabular}{|c|c|c|c|c|c|c|c|c|c|c|}
\hline \multirow[b]{2}{*}{ model } & \multicolumn{5}{|c|}{$E_{\min }=10^{17.5} \mathrm{eV}$} & \multicolumn{5}{|c|}{$E_{\min }=10^{18} \mathrm{eV}$} \\
\hline & $n$ & $\gamma$ & $\omega_{\text {cas }}{ }^{a}$ & $\delta_{I \text { best }}$ & $\delta_{I I \text { best }}$ & $n$ & $\gamma$ & $\omega_{\text {cas }}{ }^{a} \mid$ & $\delta_{I \text { best }}$ & $\delta_{I I \text { best }}$ \\
\hline & \multicolumn{10}{|c|}{ fit with Fermi-LAT bound: } \\
\hline best fit & 3.50 & 2.49 & 5.8 & 0.005 & 0 . & 3.20 & 2.52 & 5.2 & 0.050 & 0.045 \\
\hline $\min . \omega_{\text {cas }}$ & 4.50 & 2.31 & 4.4 & -0.235 & -0.245 & 2.25 & 2.47 & 1.7 & -0.120 & -0.150 \\
\hline $\max \cdot \omega_{\text {cas }}$ & 4.60 & 2.36 & 5.8 & -0.185 & -0.175 & 3.35 & 2.55 & 5.8 & 0.050 & 0.060 \\
\hline $\min . \omega_{\pi}$ & 2.00 & 2.67 & 4.9 & 0.215 & 0.235 & 2.00 & 2.51 & 1.8 & -0.070 & -0.095 \\
\hline \multirow[t]{2}{*}{$\max . \omega_{\pi}$} & 4.80 & 2.29 & 5.8 & -0.220 & -0.215 & 5.10 & 2.29 & 5.8 & -0.250 & -0.250 \\
\hline & \multicolumn{10}{|c|}{ fit without Fermi-LAT bound: } \\
\hline $\max \cdot \omega_{\text {cas }}$ & 4.45 & 2.44 & 15 & 0.135 & 0.155 & 5.25 & 2.36 & 27 & 0.205 & 0.205 \\
\hline $\max . \omega_{\pi}$ & 4.80 & 2.36 & 14 & 0.050 & 0.055 & 5.30 & 2.35 & 26 & 0.190 & 0.190 \\
\hline
\end{tabular}

\begin{tabular}{|c|c|c|c|c|c|c|c|c|c|c|}
\hline \multirow[b]{2}{*}{ model } & \multicolumn{5}{|c|}{$E_{\min }=10^{18.5} \mathrm{eV}$} & \multicolumn{5}{|c|}{$E_{\min }=10^{19} \mathrm{eV}$} \\
\hline & $n$ & $\gamma$ & $\omega_{\text {cas }}{ }^{a}$ & $\delta_{\text {Ibest }}$ & $\delta_{I I \text { best }}$ & $n$ & $\gamma$ & $\omega_{\text {cas }}{ }^{a}$ & $\delta_{I \text { best }}$ & $\delta_{I I \text { best }}$ \\
\hline & \multicolumn{10}{|c|}{ fit with Fermi-LAT bound: } \\
\hline best fit & 4.05 & 2.47 & 5.8 & 0.015 & 0.005 & 4.60 & 2.50 & 4.4 & -0.030 & -0.065 \\
\hline min. $\omega_{\text {cas }}$ & 2.00 & 2.45 & 1.4 & -0.050 & -0.060 & 2.00 & 2.88 & 0.44 & -0.220 & -0.250 \\
\hline $\max . \omega_{\text {cas }}$ & 4.95 & 2.37 & 5.8 & -0.165 & -0.160 & 4.45 & 2.13 & 5.8 & 0.130 & 0.090 \\
\hline $\min . \omega_{\pi}$ & 2.00 & 2.63 & 2.1 & 0.075 & 0.070 & 2.00 & 2.88 & 0.44 & -0.220 & -0.250 \\
\hline \multirow[t]{2}{*}{$\max . \omega_{\pi}$} & 5.35 & 2.28 & 5.8 & -0.240 & -0.250 & 4.40 & 2.10 & 5.8 & 0.145 & 0.100 \\
\hline & \multicolumn{10}{|c|}{ fit without Fermi-LAT bound: } \\
\hline $\max . \omega_{\text {cas }}$ & 6.00 & 2.49 & 30 & 0.120 & 0.135 & 6.00 & 2.14 & 23 & 0.250 & 0.210 \\
\hline $\max . \omega_{\pi}$ & 6.00 & 2.47 & 29 & 0.120 & 0.125 & 6.00 & 2.10 & 23 & 0.250 & 0.210 \\
\hline
\end{tabular}

$a_{\text {in units of } 10^{-7} \mathrm{eV} / \mathrm{cm}^{3}}$

Table 1: Cosmic ray source parameters which best fit the HiRes data [5], along with those which yield minimal and maximal contributions to $\omega_{\pi}$ (i.e. neutrino fluxes) and $\omega_{\text {cas }}=\omega_{\pi}+\omega_{\mathrm{BH}}$ (i.e. $\gamma$-ray fluxes), all at the $99 \%$ C.L.

These results are obtained assuming an energy scale uncertainty $\sigma_{E_{s}}=5 \%$ with a "top-hat" prior for the corresponding energy shifts which are taken to be uncorrelated for HiRes I and HiRes II. In Fig. 3 we explore the dependence of the results on these assumptions by using a different form for the prior, assuming the energy shifts to be correlated between the two experiments, or reducing the uncertainty to $\sigma_{E_{s}}=15 \%$. As seen in the figure, the main effect is associated with the reduction of the energy scale uncertainty which, as expected, results in a worsening of the GOF for models with larger $n$. This is directly related to the normalization constraint from Eq. 10. If one naively ignores the energy scale uncertainty, the constraint in Eq. (10) rules out models with $n \gtrsim 3$ (the precise value depending on the assumed $E_{\min }$ ). However, once the energy scale uncertainty is included, the constraint of Eq. 10 plays a weaker role on the determination of the GOF of the models. It does however imply a maximum value of $\mathcal{N}_{\text {best }}$ which, as we will see, impacts the corresponding ranges of neutrino fluxes.

The corresponding range of $\gamma$-ray and cosmogenic neutrino fluxes (summed over flavour) is shown in Fig. 4 for models with minimal and maximal energy density at the 99\% C.L. As expected, the maximum $\gamma$-ray fluxes are consistent with the Fermi-LAT data within the errors. For illustration, we also show as a dotted line the "naive" $\gamma$-ray limit $E^{2} J_{\text {cas }} \lesssim c \omega_{\text {cas }}^{\max } / 4 \pi \log (\mathrm{TeV} / \mathrm{GeV})$, corresponding to a $\gamma$-ray flux in the $\mathrm{GeV}-\mathrm{TeV}$ range which saturates the energy density 10 .

We have not included in the analysis the results from the Auger Collaboration [6, 17, which are shown 

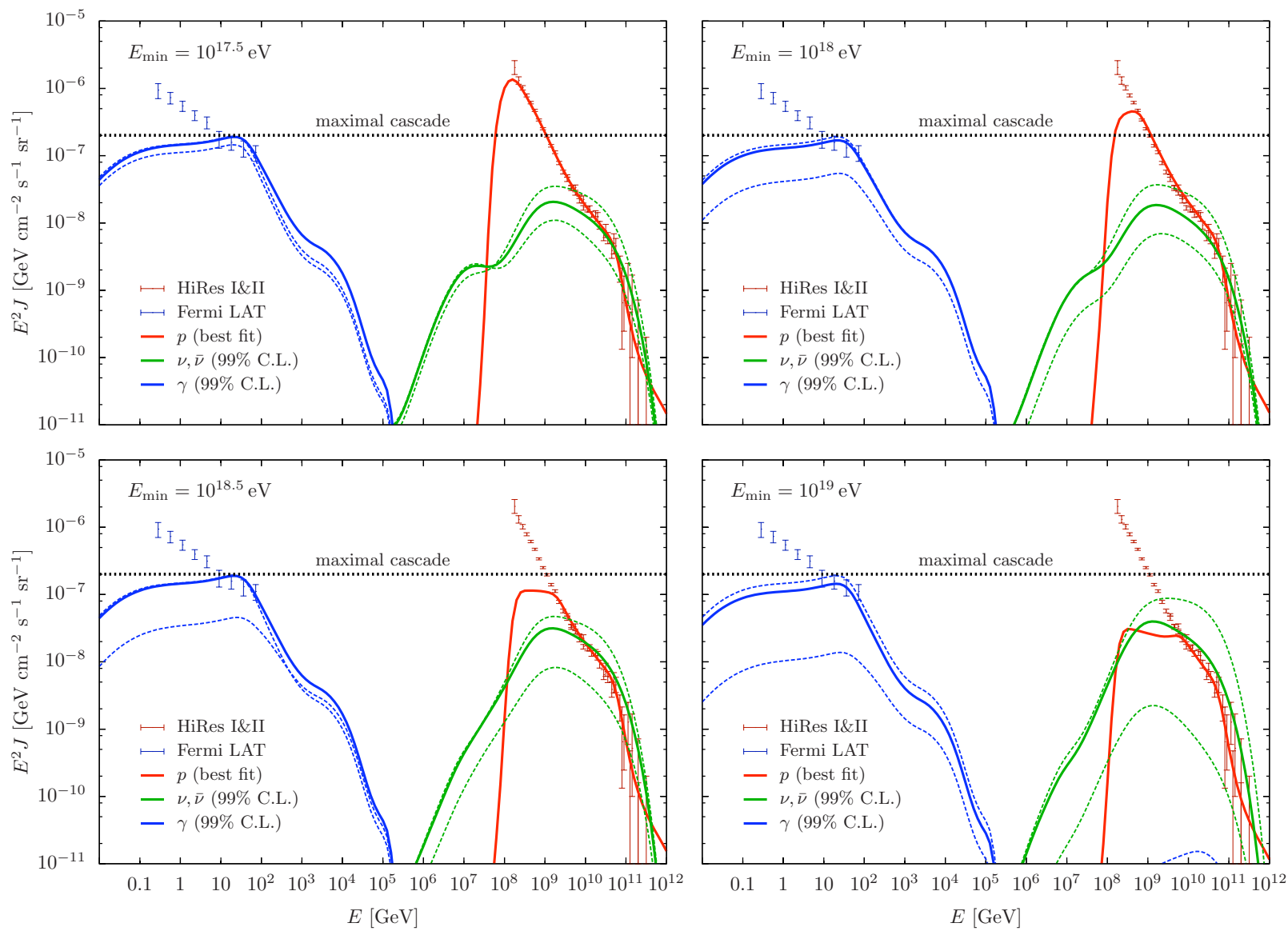

Figure 4: Comparison of proton, neutrino and $\gamma$-ray fluxes for different crossover energies. We show the best-fit values (solid lines) as well as neutrino and $\gamma$-ray fluxes within the $99 \%$ C.L. with minimal and maximal energy density (dashed lines). The values of the corresponding model parameters can be found in Table. 1 . The dotted line labeled "maximal cascade" indicates the approximate limit $E^{2} J_{\text {cas }} \lesssim c \omega_{\text {cas }}^{\max } / 4 \pi \log (\mathrm{TeV} / \mathrm{GeV})$, corresponding to a $\gamma$-ray flux in the $\mathrm{GeV}-\mathrm{TeV}$ range saturating the energy density 10). The $\gamma$-ray fluxes are marginally consistent at the $99 \%$ C.L. with the highest energy measurements by Fermi-LAT. The contribution around $100 \mathrm{GeV}$ is somewhat uncertain due to uncertainties in the cosmic infrared background.

in Fig2 2 for illustration only (hence our results are directly comparable to those in Ref.[22]). As described in Refs. 6, 17, besides the energy scale uncertainty there is also an (energy-dependent) energy resolution uncertainty which implies that bin-to-bin migrations influence the reconstruction of the flux and spectral shape. Since the form of the corresponding error matrix is not public, this data [6, 17] cannot be analysed outside the Auger Collaboration.

\section{Discussion}

The cosmogenic neutrino fluxes that we have shown in Fig. 4 are compared to present upper limits on the diffuse neutrino flux in Fig. 5. As before, the solid green line shows the neutrino flux (summed over flavours) corresponding to the best fit of the proton spectra and the dashed green line indicate the range of neutrino fluxes within the $99 \%$ C.L. For all crossover energies considered, the range of models at the $99 \%$ C.L. is consistent with existing neutrino limits. For illustration, the thin dotted line shows the larger range of neutrino fluxes at the $99 \%$ C.L. corresponding to a fit without the Fermi LAT constraint (cf. the black 

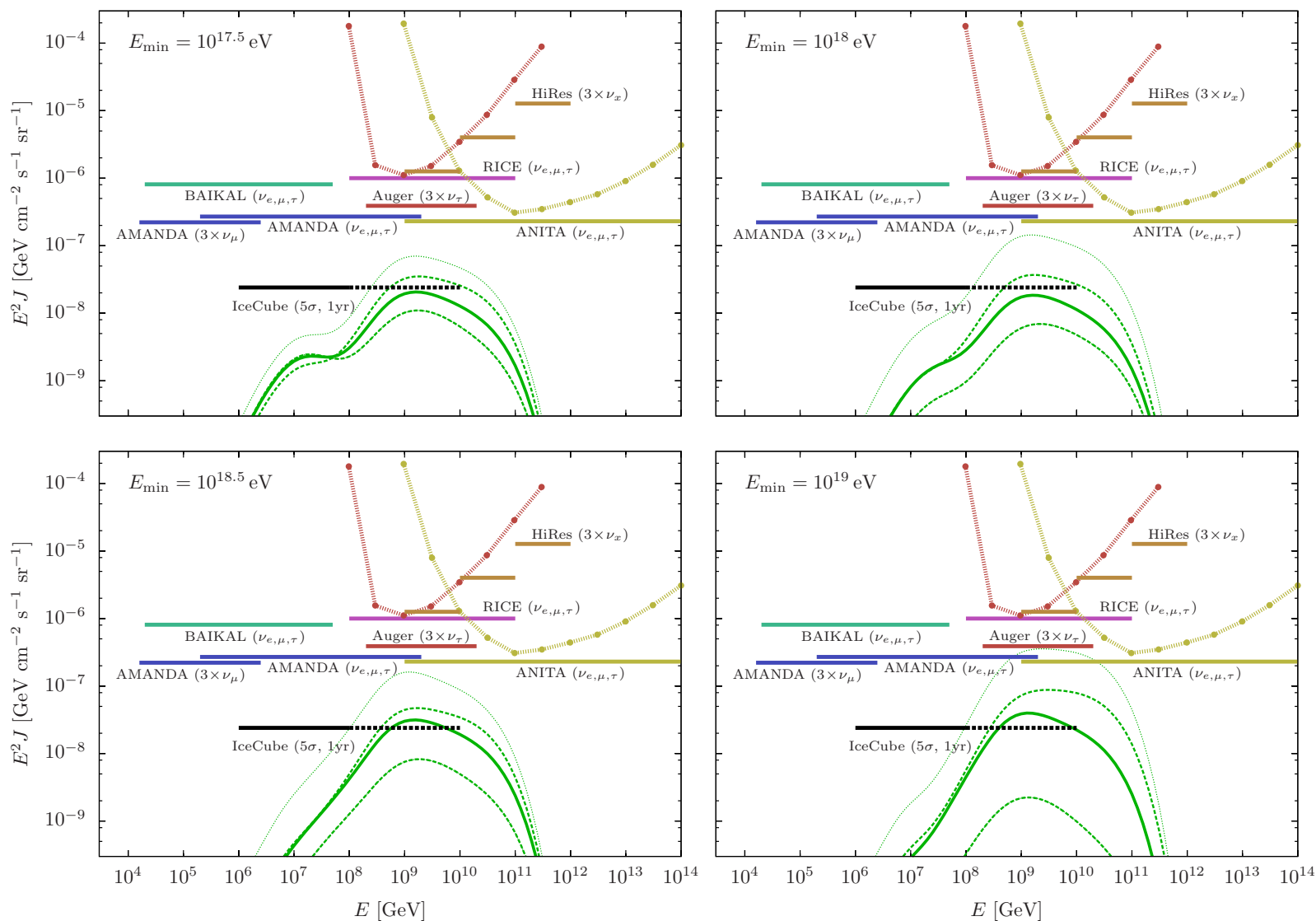

Figure 5: The predicted best fit (solid) and 99\% C.L. range of cosmogenic neutrino fluxes with (dashed) and without (dotted) the Fermi-LAT constraint. The values of the corresponding model parameters can be found in Table. 1 For comparison we show upper limits on the total diffuse neutrino flux from AMANDA 35] 36, Auger 34, Lake Baikal 37, HiRes 38. (minimum of $\nu_{\mu}$ and $\nu_{\tau}$ channel), RICE 39 and ANITA 40]. The black solid line shows the $5 \sigma$ sensitivity of IceCube after just 1 year of observation [42. The cutoff at $10^{8} \mathrm{GeV}$ is artificial so we also show an extrapolation to higher energies as a black dashed line following Ref.411). All limits are obtained assuming an equal distribution between neutrino flavours: $N_{\nu_{e}}: N_{\nu_{\mu}}: N_{\nu_{\tau}} \sim 1: 1: 1$ (and scaled appropriately where necessary). Integrated limits assuming an $E^{-2}$ spectrum are shown as solid lines and differential limits as dotted lines (both limits are shown for Auger and ANITA).

contours in the left panel of Fig. 11. It is apparent that this indirect bound from $\mathrm{GeV}$-TeV $\gamma$-rays does reduce the number of possible models significantly.

At this point it is worth stressing that the Fermi-LAT spectrum used in this analysis is not the result of a direct observation but is derived by a foreground subtraction scheme. The extra-galactic $\gamma$-ray background inferred by EGRET 44 shows a significantly larger intensity and a harder spectral index. A possible source of the differences could be due to the different diffuse galactic emission (DGE) models used in the analysis. As pointed out in 21] a re-analysis of the EGRET data with an updated DGE model 45] is comparable with the intensity observed with Fermi-LAT. It is beyond the scope of this paper to address these systematic uncertainties. The maximal effect of a larger $\gamma$-ray background intensity is indicated by the extended parameter regions shown in Fig. 1 which are derived without the Fermi-LAT constraint together with the corresponding range of neutrino fluxes in Fig. 5

The overall range of neutrino fluxes increases along with the crossover energy - not only in magnitude, which is expected already due to the reduced set of CR data used in the GOF test, but also to significantly larger neutrino fluxes. Also the cosmogenic neutrino flux of the best-fit models increases by over a factor 
of two in the peak region $\left(\sim 10^{9} \mathrm{GeV}\right)$. This confirms our earlier suspicion that an increasing value of the crossover energy allows a larger contribution of cosmogenic neutrinos relative to the $\gamma$-rays and hence larger neutrino fluxes.

Figure 5 also shows the estimated sensitivity of IceCube [43] $(5 \sigma)$ to neutrino fluxes in the $10^{6}-10^{8} \mathrm{GeV}$ (solid) 42 and the $10^{8}-10^{10} \mathrm{GeV}$ (dotted) [4] energy range after one year of observation. IceCube located at the South Pole is presently the largest neutrino telescope. On completion in early 2011 it will consist of a $\mathrm{km}^{3}$-scale detector of transparent glacial ice, that is constantly monitored for Cerenkov light emission of secondary charged particles from high energy neutrino interactions. It is apparent from Fig. 5 that IceCube's sensitivity after one year is already sufficient to probe cosmogenic neutrino fluxes from an allproton spectrum of extra-galactic cosmic rays. If the crossover energy exceeds $10^{18.5} \mathrm{eV}$, the best-fit model of the HiRes data is within reach of IceCube.

In summary we find that while the expected range of cosmogenic neutrino fluxes in all-proton models is indeed reduced due to the constraint from the diffuse $\gamma$-ray background measurements, neutrino fluxes compatible at $99 \%$ C.L with the HiRes and Fermi-LAT results can be larger than those presented in Ref. 22 . by up to factor of $\sim 30$ for the same values of $E_{\max }=10^{21} \mathrm{eV}$ and $z_{\max }=2$. In particular the allowed cosmogenic flux is still within reach of neutrino observatories like IceCube. Furthermore, our results are obtained with the simple parametrization of the source spectral emission rate in Eq. (3); larger neutrino fluxes might be allowed with a more general spectrum than a simple power-law.

One can also turn this argument around and use observation or non-observation of cosmogenic neutrinos in the near future to provide additional constraints on the composition of cosmic rays 24. We have assumed here an all-proton composition for extra-galactic cosmic rays. However, as mentioned already, the chemical composition of UHE CRs is rather uncertain and may well be dominated by heavy nuclei. In this case the limits on diffuse neutrino fluxes can still serve as a probe of the possible proton fraction in cosmic rays [24, 46] and the limits from diffuse $\gamma$-rays serve as an additional probe [47.

\section{Acknowledgments}

The authors would like to thank Andrew Taylor and the anonymous referee for helpful comments on the manuscript. This work is supported by US National Science Foundation Grant No PHY-0757598 and PHY-0653342, by the Research Foundation of SUNY at Stony Brook, the UWM Research Growth Initiative. F.H. is supported by U.S. National Science Foundation-Office of Polar Program, U.S. National Science Foundation-Physics Division, and the University of Wisconsin Alumni Research Foundation. M.C.G.-G. acknowledges further support from Spanish MICCIN grants 2007-66665-C02-01, ACI2009-1038, consolideringenio 2010 grant CSD2008-0037 and by CUR Generalitat de Catalunya grant 2009SGR502. S.S. acknowledges support by the EU Marie Curie Network "UniverseNet" (HPRN-CT-2006-035863).

\section{Appendix A. Cascade Solution}

The relevant processes with background photons contributing to the differential interaction rates $\gamma_{e e}$, $\gamma_{\gamma e}$ and $\gamma_{e \gamma}$ are inverse Compton scattering (ICS), $e^{ \pm}+\gamma_{\mathrm{bgr}} \rightarrow e^{ \pm}+\gamma$, pair production $(\mathrm{PP}), \gamma+\gamma_{\mathrm{bgr}} \rightarrow$ $e^{+}+e^{-}$, double pair production (DPP) $\gamma+\gamma_{\mathrm{bgr}} \rightarrow e^{+}+e^{-}+e^{+}+e^{-}$, and triple pair production (TPP), $e^{ \pm}+\gamma_{\text {bgr }} \rightarrow e^{ \pm}+e^{+}+e^{-}\left[25,27\right.$. The angular-averaged (differential) interaction rate, $\Gamma_{i}\left(\gamma_{i j}\right)$ is defined as

$$
\begin{gathered}
\Gamma_{i}\left(z, E_{i}\right)=\frac{1}{2} \int_{-1}^{1} \mathrm{~d} \cos \theta \int \mathrm{d} \epsilon(1-\beta \cos \theta) n_{\gamma}(z, \epsilon) \sigma_{i \gamma}^{\mathrm{tot}} \\
\gamma_{i j}\left(z, E_{i}, E_{j}\right)=\Gamma_{i}\left(z, E_{i}\right) \frac{\mathrm{d} N_{i j}}{\mathrm{~d} E_{j}}\left(E_{i}, E_{j}\right)
\end{gathered}
$$




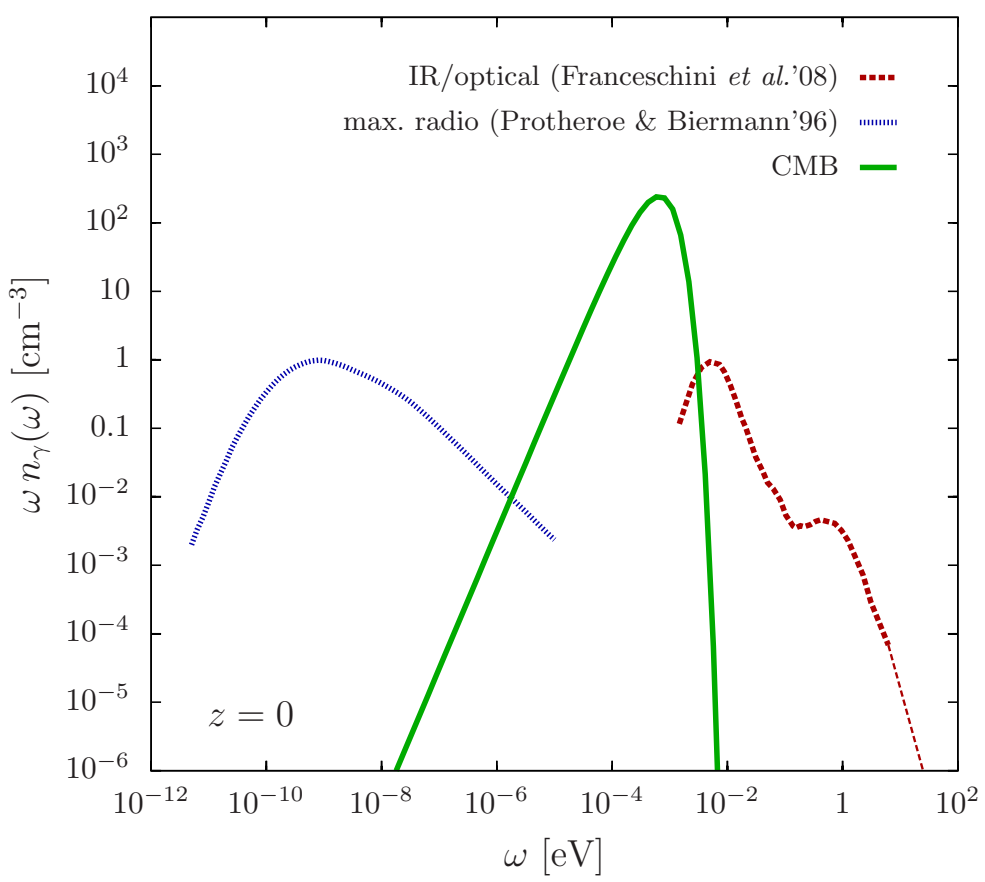

Figure A.6: The energy spectrum of the CMB 23 and the CIB in the IR/optial 48 and radio 54 range at $z=0$. The thin dashed line shows our extrapolation to UV energies.

where $n_{\gamma}(z, \epsilon)$ is the energy distribution of background photons at redshift $z$ and $\mathrm{d} N_{i j} / \mathrm{d} E_{j}$ is the angularaveraged distribution of particles $j$ after interaction of particle $i$.

Besides the contribution of the CMB the shape of the cascade spectrum depends on the cosmic infrared/optical background. We will use a recent estimate [48] (that we extrapolate slightly to UV energies as seen in Fig. A.6) and assume a redshift dependence following the star formation rate as described in Ref. 24]. This is consistent with the constraints on the $\gamma$-ray opacity of the universe set by HESS [49], MAGIC [50] and Fermi-LAT [51].

We have little direct knowledge of the cosmic radio background. An estimate made using the RAE satellite 53 is often used to calculate the cascading of UHE photons 52. A theoretical estimate has been made 54 of the intensity down to $\mathrm{kHz}$ frequencies, based on the observed luminosity function and radio spectra of normal galaxies and radio galaxies although there are large uncertainties in the assumed evolution. The calculated values are about a factor of $\sim 2$ above the measurements and to ensure maximal energy transfer in the cascade we will adopt this estimate and assume the same redshift scaling as the cosmic infrared/optical background. The magnitude of the adopted radio background radio is not important for the shape of the GeV-TeV spectrum as can be seen from Fig. 6 of Ref. [55] where even higher values are considered. We summarize the adopted cosmic radiation backgrounds in Fig. A.6.

High energetic electrons and positrons may also lose energy via synchrotron radiation in the intergalactic magnetic field $B$ with a random orientation $\sin \theta$ with respect to the velocity vector. We will assume in the following that the field strength $B$ is of $\mathcal{O}\left(10^{-12}\right) \mathrm{G}$ 32, which leads to an efficient transfer of energy into the EM cascade. The synchrotron power spectrum $\left(\mathrm{W} \mathrm{eV}^{-1}\right)$ has the form

$$
\mathcal{P}\left(E_{e}, E_{\gamma}\right)=\frac{\sqrt{3} \alpha}{2 \pi} \frac{e B \sin \theta}{m_{e}} F\left(E_{\gamma} / E_{\mathrm{c}}\right) ; \quad F(t) \equiv t \int_{t}^{\infty} \mathrm{d} z K_{5 / 3}(z),
$$

where we follow the notation of Ref. [56] with $E_{\mathrm{c}}=\left(3 e B \sin \theta / 2 m_{e}\right)\left(E_{e} / m_{e}\right)^{2}$. This can be treated as a 
continuous energy loss of the electrons and positrons with a parameter 4

$$
b_{\text {syn }}\left(E_{e}\right)=\frac{1}{2} \int \mathrm{d} \cos \theta \int \mathrm{d} E_{\gamma} \mathcal{P}\left(E_{e}, E_{\gamma}\right)=\frac{4 \alpha}{9}\left(\frac{e B}{m_{e}}\right)^{2}\left(\frac{E_{e}}{m_{e}}\right)^{2} .
$$

We will assume in the calculation that the intergalactic magnetic field is primordial with a (flux-conserving) redshift dependence $B(z)=(1+z)^{2} B(0)$. Note, that the synchrotron energy loss has then a redshift dependence similar to $\mathrm{BH}$ pair production in the $\mathrm{CMB}$, i.e. $b_{\mathrm{syn}}(z, E)=(1+z)^{2} b_{\mathrm{syn}}(0,(1+z) E)$. It is also convenient to define $\gamma_{e \gamma}^{\mathrm{syn}}\left(E_{e}, E_{\gamma}\right) \equiv \mathcal{P}\left(E_{e}, E_{\gamma}\right) / E_{\gamma}$, which has an analogous redshift dependence i.e. $\gamma_{e \gamma}^{\mathrm{syn}}\left(z, E_{e}, E_{\gamma}\right)=(1+z)^{4} \gamma_{e \gamma}^{\mathrm{syn}}\left(0,(1+z) E_{e},(1+z) E_{\gamma}\right)$.

The fast evolution of the cascade is governed by the set of differential equations,

$$
\begin{aligned}
& \partial_{\hat{t}} Y_{\gamma}(E)=-\Gamma_{\gamma}(E) Y_{\gamma}(E)+\int \mathrm{d} E^{\prime} \frac{\mathcal{P}\left(E^{\prime}, E\right)}{E} Y_{e}\left(E^{\prime}\right)+\int \mathrm{d} E^{\prime} \gamma_{e \gamma}\left(E^{\prime}, E\right) Y_{e}\left(E^{\prime}\right), \\
& \partial_{\hat{t}} Y_{e}(E)=-\Gamma_{e}(E) Y_{e}(E)+\partial_{E}\left(b(E) Y_{e}(E)\right)+\int \mathrm{d} E^{\prime}\left[\gamma_{\gamma e}\left(E^{\prime}, E\right) Y_{\gamma}\left(E^{\prime}\right)+\gamma_{e e}\left(E^{\prime}, E\right) Y_{e}\left(E^{\prime}\right)\right],
\end{aligned}
$$

which determines the evolution on short time-scales $\Delta \hat{t} \Gamma_{p \gamma} \ll 1$ (the redshift $z$ is kept fixed meanwhile). The initial condition $\left.Y_{\gamma / e}(E)\right|_{\hat{t}=0}$ is given by the sum of previously developed cascades and the newly generated contributions from proton interactions.

The solution of Eqs. A.5 and A.6 for an infinitesimally small step $\Delta \hat{t}$ can be written for a discrete energy spectrum, $N_{i} \simeq \Delta \bar{E}_{i} Y_{i}$, as

$$
\left(\begin{array}{l}
N_{\gamma} \\
N_{e}
\end{array}\right)_{i}(\hat{t}+\Delta \hat{t}) \simeq \sum_{j}\left(\begin{array}{ll}
T_{\gamma \gamma}(\Delta \hat{t}) & T_{e \gamma}(\Delta \hat{t}) \\
T_{\gamma e}(\Delta \hat{t}) & T_{e e}(\Delta \hat{t})
\end{array}\right)_{j i}\left(\begin{array}{l}
N_{\gamma} \\
N_{e}
\end{array}\right)_{j}(\hat{t})
$$

With the transition matrix $\mathcal{T}(\Delta \hat{t})$, defined by Eq. A.7), we can efficiently follow the development of the EM cascade over a distance $\Delta t=2^{N} \Delta \hat{t}$ via matrix doubling [33]:

$$
\mathcal{T}\left(2^{N} \Delta \hat{t}\right) \simeq[\mathcal{T}(\Delta \hat{t})]^{N+1}
$$

We will compare our calculation with results from other investigations in Appendix Appendix C

\section{Appendix B. Energy Density of the Cascade}

We can express the system of partial integro-differential equations (1) as,

$$
\dot{Z}_{i}=\partial_{\mathcal{E}}\left(b_{i}(z, \mathcal{E}) Z_{i}(z, E)\right)-\Gamma_{i}(z, \mathcal{E}) Z_{i}+(1+z) \mathcal{L}_{i}^{\mathrm{eff}}(z, \mathcal{E}),
$$

where we have defined $\mathcal{E}=(1+z) E$, and $Z_{i}(z, E) \equiv(1+z) Y_{i}(z, \mathcal{E})$, subject to the boundary condition $Z_{j}\left(z_{\max }, E\right)=0$. The effective source term in Eq. (B.1) is

$$
\mathcal{L}_{i}^{\text {eff }}=\mathcal{L}_{i}+\sum_{j} \int \mathrm{d} E_{j} \gamma_{j i}\left(z, \mathcal{E}_{j}, E_{i}\right) Z_{j}
$$

The total energy of the cascade is given in Eq. (6). This can be obtained by integrating Eq. (B.1):

$$
\frac{\mathrm{d}}{\mathrm{d} t}\left[\int \mathrm{d} E E Z_{\mathrm{cas}}(z, E)\right]=-\int \mathrm{d} E E \partial_{\mathcal{E}}\left[b_{\mathrm{cas}}(z, \mathcal{E}) Z_{\mathrm{p}}(z, E)\right] .
$$

\footnotetext{
${ }^{4}$ Note, the identity $\int \mathrm{d} E\left[E \partial_{E}\left(b n_{e}\right)+\int \mathrm{d} E^{\prime} \mathcal{P}\left(E^{\prime}, E\right) n_{e}\right]=0$, implying overall energy conservation.
} 

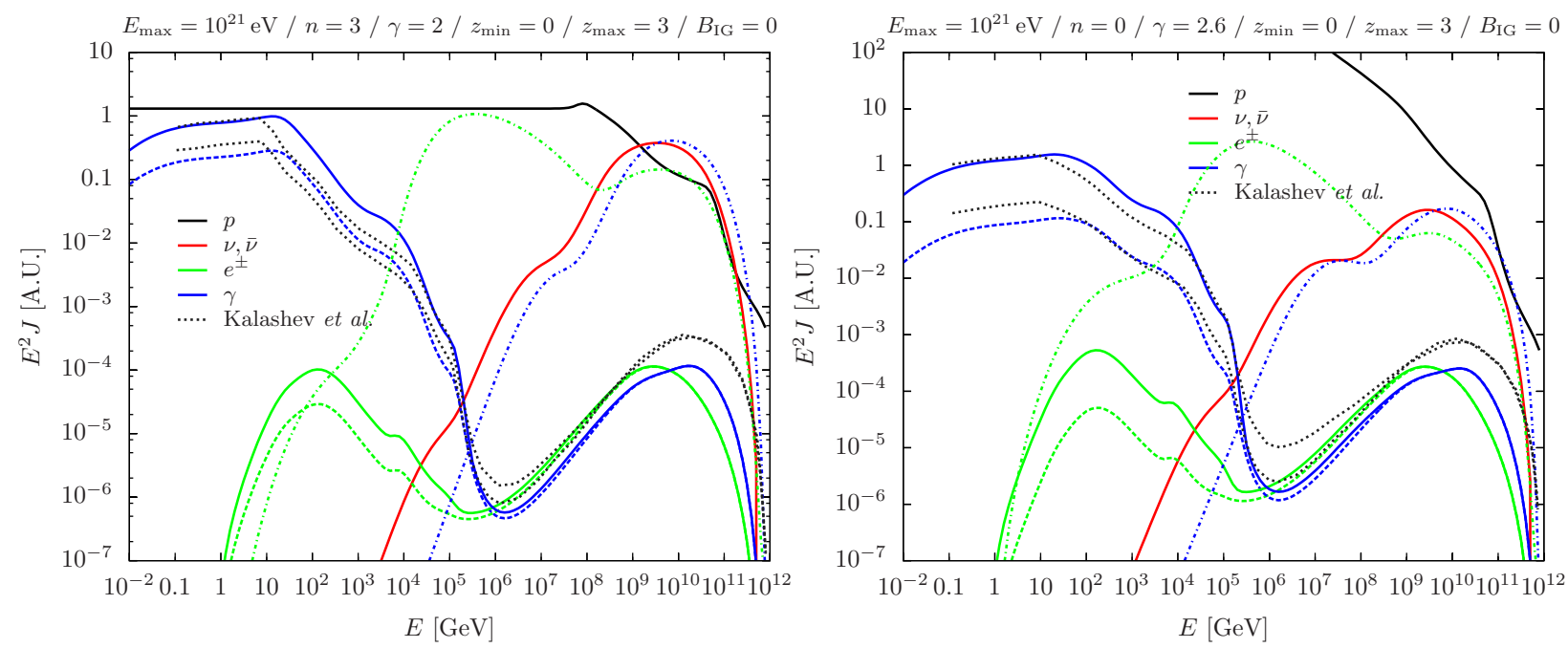

Figure B.7: Comparison of our calculations with the $\gamma$-ray spectra of Ref. [57. shown as black doted lines. We normalize the $\gamma$-ray (green) and electron/positron (red) spectra to the proton spectra (black solid line). For comparison, the dashed lines show the spectra without the contribution of BH pairs and the dashed-dotted lines show the spectra without EM cascades.

Integrating the r.h.s. by parts yields

$$
\text { r.h.s. }=-\int \mathrm{d} E \partial_{E}\left[E \frac{1}{1+z} b_{\text {cas }}(z, \mathcal{E}) Z_{\mathrm{p}}(z, E)\right]+\int \mathrm{d} E \frac{1}{1+z} b_{\text {cas }}(z, \mathcal{E}) Z_{\mathrm{p}}(z, E) .
$$

The first term vanishes since $b_{\text {cas }}=0$ for sufficiently low energies and $Z_{\mathrm{p}}=0$ beyond the maximal energy. The time integration of the l.h.s. between the present epoch $(t=0)$ and the first sources $\left(t_{\max }\right)$ gives

$$
\int_{0}^{t_{\max }} \mathrm{d} t[\text { l.h.s. }]=\int \mathrm{d} E E n_{\text {cas }}(E)=\omega_{\text {cas }},
$$

hence we obtain Eq. (6).

\section{Appendix C. Comparison of Gamma-Ray Spectra}

Figure B.7 compares our calculations with spectra derived in Ref. [57] (Figs. 1 and 2) using $n=3$ and $\gamma=2$ (left plot) as well as $n=0$ and $\gamma=2.6$ (right plot), respectively, with (upper) and without (lower) $\mathrm{BH}$ contributions.

The $\gamma$-ray spectra without BH pairs in the cascade are consistent with Ref. [57] apart from a slightly smaller energy density (recognizable as an overall shift downwards) probably due to the difference in the adopted IR/optical background. Since the energy density derived from our spectra agrees with the value obtained from Eq. (6) within 10\%, we believe that the overall normalization of our spectra is correct.

For a full calculation, i.e. including the BH pairs, our overall energy density agrees well with the calculation in Ref. [57. Again, the energy density derived from our spectra agrees with the value obtained from Eq. (6) within $10 \%$. The pair production dip at $10^{6} \mathrm{GeV}$ is more pronounced in our spectra. Note that the energy loss of the cascade beyond $10^{5} \mathrm{GeV}$ is much more rapid than the Bethe-Heitler pair production rate, hence the modest increase in $\gamma$-rays beyond this energy meets our expectations. Moreover, instead of using a power-law approximation $\left(\mathrm{d} n / \mathrm{d} E_{ \pm} \propto E_{ \pm}^{-7 / 4}\right)$ for the $\mathrm{BH} e^{ \pm}$spectrum (see Ref.[58] for a critical discussion) we use the exact differential cross-section of Ref. 25]. 

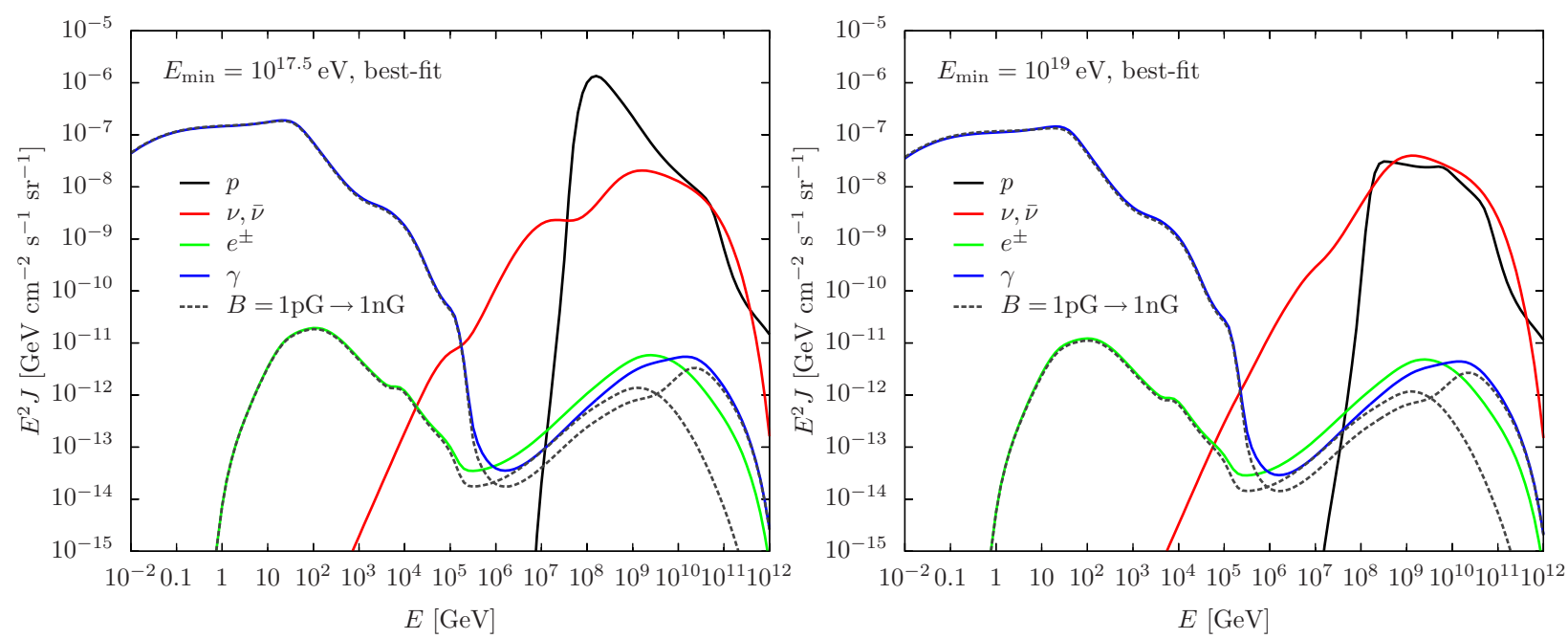

Figure C.8: The effect of a large intergalactic magnetic field. The solid lines show the spectra of the best-fit for crossover energies $E_{\mathrm{min}}=10^{17.5} \mathrm{eV}$ (left) and $10^{19} \mathrm{eV}$ (right) using $B_{\mathrm{IG}}=10^{-12} \mathrm{G}$. The dashed lines show the corresponding results for a much larger field strength $B_{\mathrm{IG}}=10^{-9} \mathrm{G}$. The $\gamma$-ray flux in the GeV-TeV region relevant for the Fermi-LAT spectrum is practically unaffected.

\section{References}

[1] A. A. Penzias and R. W. Wilson, Astrophys. J. 142, 419 (1965).

[2] K. Greisen, Phys. Rev. Lett. 16, 748 (1966).

[3] G. T. Zatsepin and V. A. Kuzmin, JETP Lett. 4, 78 (1966) [Pisma Zh. Eksp. Teor. Fiz. 4, 114 (1966)].

[4] V. S. Beresinsky and G. T. Zatsepin, Phys. Lett. B 28, 423 (1969).

[5] R. Abbasi et al. [HiRes Collaboration], Phys. Rev. Lett. 100, 101101 (2008) arXiv:astro-ph/0703099].

[6] J. Abraham et al. [Pierre Auger Collaboration], Phys. Rev. Lett. 101, 061101 (2008) arXiv:0806.4302 [astro-ph]].

[7] F. W. Stecker, Astrophys. J. 228, 919 (1979).

[8] S. Yoshida and M. Teshima, Prog. Theor. Phys. 89, 833 (1993); R. J. Protheroe and P. A. Johnson, Astropart. Phys. 4, 253 (1996) arXiv:astro-ph/9506119; R. Engel, D. Seckel and T. Stanev, Phys. Rev. D 64, 093010 (2001) arXiv:astro$\mathrm{ph} / 0101216$.

[9] C. T. Hill and D. N. Schramm, Phys. Rev. D 31, 564 (1985).

[10] J. Linsley, Proceedings of ICRC 1963, Jaipur, India, pp. 77-99

[11] C. T. Hill and D. N. Schramm, Phys. Rev. D 31, 564 (1985).

[12] T. Wibig and A. W. Wolfendale, J. Phys. G 31, 255 (2005). arXiv:astro-ph/0410624.

[13] V. Berezinsky, A. Z. Gazizov and S. I. Grigorieva, Phys. Rev. D 74, 043005 (2006). arXiv:hep-ph/0204357.

14] Z. Fodor, S. D. Katz, A. Ringwald and H. Tu, JCAP 0311, 015 (2003) arXiv:hep-ph/0309171.

[15] D. Hooper, A. Taylor and S. Sarkar, Astropart. Phys. 23, 11 (2005) arXiv:astro-ph/0407618; M. Ave, N. Busca, A. V. Olinto, A. A. Watson and T. Yamamoto, Astropart. Phys. 23, 19 (2005) arXiv:astro-ph/0409316; D. Allard et al., JCAP 0609, 005 (2006) arXiv:astro-ph/0605327.

[16] L. A. Anchordoqui, H. Goldberg, D. Hooper, S. Sarkar and A. M. Taylor, Phys. Rev. D 76, 123008 (2007) arXiv:0709.0734 [astro-ph]]. We wish to stress that the essential results of this analysis are not altered by the new Auger data [17, 18.

[17] J. Abraham et al. [Pierre Auger Collaboration], Phys. Lett. B 685, 239 (2010) arXiv:1002.1975 [astro-ph.HE]].

[18] J. Abraham et al. [Pierre Auger Collaboration], Phys. Rev. Lett. 104, 091101 (2010) arXiv:1002.0699 [astro-ph.HE]].

[19] T. Wibig, arXiv:0810.5281 [hep-ph].

[20] R. Ulrich, R. Engel, S. Muller, F. Schussler and M. Unger, Nucl. Phys. Proc. Suppl. 196, 335 (2009) arXiv:0906.3075 [astro-ph.HE]].

[21] A. A. Abdo et al. [Fermi-LAT Collaboration], Phys. Rev. Lett. 104, 101101 (2010) arXiv:1002.3603 [astro-ph.HE]].

[22] V. Berezinsky, A. Gazizov, M. Kachelriess and S. Ostapchenko, arXiv:1003.1496 [astro-ph.HE].

[23] C. Amsler et al. [Particle Data Group], Phys. Lett. B 667, 1 (2008).

[24] M. Ahlers, L. A. Anchordoqui and S. Sarkar, Phys. Rev. D 79, 083009 (2009) arXiv:0902.3993 [astro-ph.HE]].

[25] G. R. Blumenthal, Phys. Rev. D 1, 1596 (1970).

[26] A. Mücke, R. Engel, J. P. Rachen, R. J. Protheroe and T. Stanev, Comput. Phys. Commun. 124, 290 (2000) arXiv:astroph/9903478.

[27] G. R. Blumenthal and R. J. Gould, Rev. Mod. Phys. 42, 237 (1970)

[28] S. Lee, Phys. Rev. D 58, 043004 (1998) arXiv:astro-ph/9604098.

[29] S. V. Demidov and O. E. Kalashev, J. Exp. Theor. Phys. 108, 764 (2009) arXiv:0812.0859 [astro-ph]].

[30] P. P. Kronberg, Rept. Prog. Phys. 57, 325 (1994). 
[31] J. Wdowczyk, W. Tkaczyk and A. W. Wolfendale, J. of Phys. A 5, 1419-1432 (1972).

[32] K. Dolag, D. Grasso, V. Springel and I. Tkachev, JCAP 0501, 009 (2005) arXiv:astro-ph/0410419.

[33] R. J. Protheroe and T. Stanev, Mon. Not. R. Astron. Soc. 264, 191 (1993).

[34] J. Abraham et al. [Pierre Auger Collaboration], Phys. Rev. D 79, 102001 (2009) arXiv:0903.3385 [astro-ph.HE]]; Phys. Rev. Lett. 100, 211101 (2008) arXiv:0712.1909 [astro-ph]].

[35] A. Achterberg et al. [IceCube Collaboration], Phys. Rev. D 76, 042008 (2007) [Erratum-ibid. D 77, 089904 (2008)] arXiv:0705.1315 [astro-ph]].

[36] M. Ackermann et al. [IceCube Collaboration], Astrophys. J. 675, 1014 (2008) arXiv:0711.3022 [astro-ph]].

[37] V. Aynutdinov et al. [BAIKAL Collaboration], Astropart. Phys. 25, 140 (2006) arXiv:astro-ph/0508675].

[38] K. Martens [HiRes Collaboration], arXiv:0707.4417 [astro-ph].

[39] I. Kravchenko et al., Phys. Rev. D 73, 082002 (2006) arXiv:astro-ph/0601148.

[40] S. W. Barwick et al. [ANITA Collaboration], Phys. Rev. Lett. 96, 171101 (2006) arXiv:astro-ph/0512265].

[41] F. Halzen and D. Hooper, Phys. Rev. Lett. 97, 099901 (2006) arXiv:astro-ph/0605103.

[42] A. Achterberg et al. [IceCube Collaboration], Astropart. Phys. 26, 155 (2006).

[43] J. Ahrens et al. [IceCube Collaboration], Astropart. Phys. 20, 507 (2004) arXiv:astro-ph/0305196].

[44] P. Sreekumar et al. [EGRET Collaboration], Astrophys. J. 494, 523 (1998) arXiv:astro-ph/9709257.

[45] A. W. Strong, I. V. Moskalenko and O. Reimer, Astrophys. J. 613, 956 (2004) arXiv:astro-ph/0405441].

[46] L. A. Anchordoqui and T. Montaruli arXiv:0912.1035 [astro-ph.HE]. See Fig. 13 for an updated analysis of [24].

[47] M. Ahlers et al., in preparation

[48] A. Franceschini, G. Rodighiero and M. Vaccari, Astron. Astrophys. 487, 837 (2008) arXiv:0805.1841 [astro-ph]]

[49] F. Aharonian et al. [H.E.S.S. Collaboration], Nature 440, 1018 (2006) arXiv:astro-ph/0508073.

[50] E. Aliu et al. [MAGIC Collaboration], Science 320, 1752 (2008) arXiv:0807.2822 [astro-ph]].

[51] A. A. Abdo et al. [Fermi-LAT Collaboration], arXiv:1005.0996 [astro-ph.HE].

[52] P. Bhattacharjee and G. Sigl, Phys. Rept. 327, 109 (2000) arXiv:astro-ph/9811011].

[53] T. A. Clark, L. W. Brown, and J. K. Alexander, Nature 228, 847 (1970).

[54] R. J. Protheroe and P. L. Biermann, Astropart. Phys. 6, 45 (1996) [Erratum-ibid. 7, 181 (1996)], arXiv:astro-ph/9605119].

[55] S. Sarkar, Acta Phys. Polon. B 35, 351 (2004) arXiv:hep-ph/0312223.

[56] R. Schlickeiser, "Cosmic ray astrophysics," Berlin, Germany: Springer (2002) $519 p$

[57] O. E. Kalashev, D. V. Semikoz and G. Sigl, Phys. Rev. D 79, 063005 (2009) arXiv:0704.2463 [astro-ph]].

[58] S. R. Kelner and F. A. Aharonian, Phys. Rev. D 78, 034013 (2008) arXiv:0803.0688 [astro-ph]]. 